\title{
An Overview of Children's Oral Health-Related Quality of Life Assessment: From Scale Development to Measuring Outcomes
}

\author{
M.W. Genderson ${ }^{a} \quad$ L. Sischo ${ }^{b} \quad$ K. Markowitz ${ }^{c} \quad$ D. Fine ${ }^{c} \quad$ H.L. Broder ${ }^{b}$

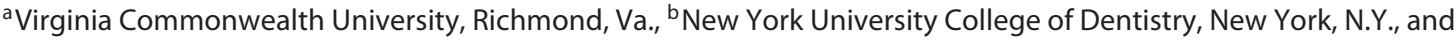 \\ 'University of Medicine and Dentistry of New Jersey, Newark, N.J., USA
}

\section{Key Words}

Clinically meaningful difference · Dental caries · Minimally important difference - Oral health-related quality of life . Quality of life

\begin{abstract}
The objectives of this paper are to present an overview of children's oral health-related quality of life and include specific applications for using quality of life assessment in dental research. The process of developing pediatric oral healthrelated quality of life measures, in particular the Child Oral Health Impact Profile, is outlined. Examples of children's oral health-related quality of life measurement in caries research are also provided. Quality of life outcomes are presented and discussed in the context of caries research. Lastly, the relevance of measuring clinically meaningful difference in the context of measuring outcomes research is highlighted with recommendations for future research. @ 2013 S. Karger AG, Basel
\end{abstract}

Many health service researchers today embrace a concept of health that goes beyond the absence of disease to include 'a complete state of physical, mental, and social well-being' [WHO, 1948]. In focusing on health as a mul- tidimensional concept that incorporates symptoms, physical functioning, and emotional and social well-being, these researchers incorporate quality of life (QoL) and oral health-related quality of life (OHRQoL) into their biopsychosocial health model. QoL, or 'individuals' perceptions of their position in life in the context of the culture and value systems in which they live, and in relation to their goals, expectations, standards, and concerns' [WHOQOL, 1995], is now recognized as a valid parameter in patient assessment in nearly every area of physical and mental healthcare, including oral health. Indeed, several instruments now exist to measure OHRQoL, a multidimensional construct that includes a subjective evaluation of an individual's oral health, functional well-being, emotional well-being, expectations and satisfaction with care, and sense of self [Sischo and Broder, 2011a].

\section{Why Is OHRQoL Important and How Can It Be Used in Research?}

OHRQoL plays an important role in understanding subjective patient evaluations of and experience with oral healthcare. The subjective evaluation of OHRQoL 'reflects people's comfort when eating, sleeping and engaging in social interaction; their self-esteem; and their satis-

\section{KARGER}

E-Mail karger@karger.com

www.karger.com/cre
(C) 2013 S. Karger AG, Basel

0008-6568/13/0477-0013\$38.00/0
Hillary Broder

New York University College of Dentistry

345 E. 24th Street

New York, NY 10010 (USA)

E-Mail hillary.broder@nyu.edu 
faction with respect to their oral health' [DHHS, 2000]. Consonant with the biopsychosocial model of health, OHRQoL is the result of an interaction between oral health conditions, social and contextual factors [Locker et al., 2005], and the rest of the body [Atchison et al., 2006].

Incorporating OHRQoL creates a shift from traditional medical/dental criteria to assessment and care that focus on a person's social and emotional experience and physical functioning. Thus OHRQoL evaluations have the potential to enhance evaluation, clinical research and care in a number of ways including needs assessment of a population or a specific clinical group. For example, a recent study among children with orofacial clefts found that individuals without health insurance and those representing ethnic minorities had lower OHRQoL and a higher rate of unmet surgical needs than their privately insured counterparts [Broder et al., 2012a]. Here it is shown that the patient's perspective captured via OHRQoL assessments can illuminate health disparities among vulnerable youth with orofacial clefts. This information can allow researchers and clinicians to better define appropriate treatment goals and outcomes resulting in important benefits for individual patients, community-based dental practices, clinical research, and potentially public health policy.

Another way OHRQoL can be used is to inform and refine care by incorporating such assessments into oral health service planning for discrete populations like school-aged children. In this application, the Child Oral Impacts on Daily Performances was used as an indicator to prioritize children's sociodental needs among 11- to 12 -year-old children in Thailand [Gherunpong et al., 2006].

OHRQoL assessment can also be used as an outcome or evaluative measure across specialty areas, including pediatric caries research. OHRQoL provides a unique perspective on dental care from the child participant or from an observer like a parent. Traditional 'objective' criteria such as dental decay, missing teeth, and filled teeth (DMFT) fail to include subjective assessments (i.e., patient satisfaction, symptom reduction, and increased functional and emotional well-being) from the patient. Taking OHRQoL impacts into account, however, can differentiate need and help prioritize care for vulnerable populations [Gherunpong et al., 2006]. This information is important as most studies indicate a modest yet significant correlation between unmet needs like dental decay and children's OHRQoL.

\section{Developing Child OHRQoL Measures}

Specific issues arise when developing OHRQoL measures for children. Because oral health is 'strongly agedependent' [John et al., 2004], it is not surprising that differences in OHRQoL have been found between children and adults [Tapsoba et al., 2000]. While many instruments exist to measure adult OHRQoL designing instruments specifically for children and adolescents allows researchers to identify and examine OHRQoL factors that are unique to these populations (i.e., self-image, social acceptance, and school environment) [McGrath et al., 2004; Rumsey and Harcourt, 2004; Broder, 2007].

Several validated instruments currently exist to measure children's OHRQoL, including the Child Perception Questionnaire, the Early Childhood Oral Health Impact Score, Pediatric Oral Health-Related Quality of Life, Child Oral Impacts on Daily Performances, and the Child Oral Health Impact Profile (COHIP; see table 1 for an overview of these instruments).

The Child Perception Questionnaire was the first instrument specifically designed to measure children's OHRQoL [Locker et al., 2005]. Two age forms of the Child Perception Questionnaire currently exist - one for 8- to 10-year-olds and another for 11- to 14-year-olds. It is not clear, however, whether the two measures are continuous and can be used in longitudinal studies when children age out of the younger version. The Early Childhood Oral Health Impact Score [Pahel et al., 2007], which uses caregiver reports for proxies, is targeted for preschool children who can have a variety of dental, orthodontic, and craniofacial conditions. The Pediatric Oral Health-Related Quality of Life is a relatively new OHRQoL instrument for preschool and school-aged children. While it shows promise for inclusion in OHRQoL research, particularly in its ability to capture the impact of oral conditions like caries on both general and vulnerable populations [Huntington et al., 2011], it has yet to undergo testing for its evaluative properties in a longitudinal study. The Child Oral Impacts on Daily Performances was originally developed to measure OHRQoL in 12-year-old Thai children [Gherunpong et al., 2004], although it has since been validated in a number of languages and has undergone extensive psychometric assessments. It focuses on the negative impact of oral conditions on daily performances and has been used in several population-based studies [Yusuf et al., 2006]. The COHIP is the first children's OHRQoL instrument to incorporate both positive and negative health impacts [Sischo and Broder, 2011a], therefore has the potential to DOI: $10.1159 / 000351693$
Genderson/Sischo/Markowitz/Fine/ Broder 
Table 1. Child OHRQoL measures

\begin{tabular}{|c|c|c|c|c|}
\hline Instrument & $\begin{array}{l}\text { Date of } \\
\text { creation }\end{array}$ & $\begin{array}{l}\text { Age } \\
\text { range }\end{array}$ & $\begin{array}{l}\text { Items, } \\
\mathrm{n}\end{array}$ & $\begin{array}{l}\text { Short } \\
\text { form }\end{array}$ \\
\hline $\begin{array}{l}\text { Child Perceptions } \\
\text { Questionnaire }(11-14) \\
\text { [Jokovic et al., 2002] }\end{array}$ & 2002 & $11-14$ & 37 & $\begin{array}{l}\text { yes } \\
(16 \text { items }) \\
(8 \text { items })\end{array}$ \\
\hline $\begin{array}{l}\text { Child Perceptions } \\
\text { Questionnaire }(8-10) \\
\text { [Jokovic et al., 2002] }\end{array}$ & 2004 & $8-10$ & 25 & no \\
\hline $\begin{array}{l}\text { Child Oral Impacts on Daily Performances } \\
\text { [Gherunpong et al., 2004; Yusuf et al., 2006] }\end{array}$ & 2004 & $10-12$ & 8 & no \\
\hline $\begin{array}{l}\text { Early Childhood Oral Health Impact Scale } \\
\text { [Pahel et al., 2007] }\end{array}$ & 2007 & $3-5$ & 13 & no \\
\hline $\begin{array}{l}\text { Child Oral Health Impact Profile (COHIP) } \\
\text { [Broder et al., 2012b] }\end{array}$ & 2007 & $7-18$ & 34 & $\begin{array}{l}\text { yes } \\
\text { (19 items) }\end{array}$ \\
\hline $\begin{array}{l}\text { Pediatric Oral Health-Related Quality of Life } \\
\text { [Huntington et al., 2011] }\end{array}$ & 2011 & $2-12$ & 20 & no \\
\hline
\end{tabular}

measure more than the absence of a condition but can measure positive attributes or enhanced well-being (e.g., confidence) as a result of care. Three versions of the COHIP (child, caregiver, and teacher) are currently being used in an ongoing multicenter, longitudinal study of children's QoL [Broder, pers. commun.]. Additionally, a short form (19 items) of the COHIP has recently been validated. Short forms are quicker to administer and therefore facilitate utilizing QoL assessment in clinical studies [Broder et al., 2012b]. All of these instruments have undergone forward and reverse translation and are available in multiple languages.

The overall goal in OHRQoL instrument development can vary depending on the research goal. For example, a discriminative instrument should not contain questions to which all respondents with the disease would respond in a like manner (e.g., items to which virtually all or none of the respondents answer similarly should be deleted). Items that are strongly influenced by factors other than the disease of interest should be excluded. Including items that correlate with one another will ensure high internal consistency, which is important for sound statistical analysis. On the other hand, an evaluative tool for clinical trials should have test items that are sensitive to change as a result of treatment.

Another consideration for measurement development and selection involves change in health in the context of treatment, a phenomenon which is referred to as response shift. Response shift encompasses changes within people regarding internal standards, values, or conceptualization of health-related QoL, particularly when health state changes. It can also affect standard psychometric indices, such as reliability and validity [Schwartz, 2010]. Acknowledging and assessing the degree to which response shifts do occur in the context of oral health and oral health treatment [Gregory et al., 2005] can inform development of measures designed to reveal when it may be occurring. This is particularly an important consideration when evaluating OHRQoL for groups known to have a lengthy and often complicated treatment such as adults with edentulism [Kimura et al., 2012], people with disabilities, and youth with extensive oral health care needs [Reissmann et al., 2012].

Regardless of the application, items must be clear and relevant to the target group and calibrated to the appropriate reading level for the targeted age group using an established methodology (e.g., Flesch-Kincaid). Length and formatting of a questionnaire (e.g., line shading) can also be critical, especially for children, in order to reduce error and fatigue [Schwartz, 2010].

Achieving these goals requires that questionnaire development be an iterative process that includes a literature review, item generation, face and content validity testing, item-impact analyses, qualitative interviewing as well as consideration of theoretical issues used to nominate items for inclusion and elimination [Broder et al., 2007]. Guyatt 
et al. [1989, 1996] and Christie et al. [1993] have written extensively on this process, and more about how this multistage process was used in the development of the COHIP can be found elsewhere [Broder and Wilson-Genderson, 2007; Broder et al., 2007, 2012b]. Validity and reliability testing must be reported to demonstrate psychometric worthiness of the instrument. Scale reliability, which was measured using Cronbach's alpha coefficient, was excellent ( 0.91 for the overall score) as was the test-retest reliability of the Overall COHIP (ICC $=0.84)$ computed for a subset of participants who did not report a health change. Discriminant and convergent validity were also supported by the comparisons between and associations within the four groups of children. Effective QoL measures should reveal covariation with the severity of dental disease - thus demonstrating sensitivity within a disease group (e.g., dental caries) [Yusuf et al., 2006].

\section{OHRQoL in Pediatric Caries Research}

To demonstrate the value OHRQoL can bring to caries research, we recently analyzed OHRQoL COHIP data from a sample of underserved pediatric patients who were being evaluated/treated for caries as part of a larger study [Markowitz et al., 2012].

The sample included 102 schoolchildren from Newark, N.J. The participants assented to participate and the caregivers gave informed consent in accordance with IRB regulations. The clinical exam was performed in a mobile van donated by the Colgate-Palmolive Company using methods and procedures that have been vigorously calibrated and tested [Fine et al., 2007].

Demographics collected included age, gender, ethnicity and grade in school. A dental examination was performed and the presence of decayed, missing, and filled surfaces (DMFS) was entered on the patient's chart and these data were entered into an electronic database. In these examinations, visual examination and the dental explorer were used to detect caries. In performing tactile examination, the explorer was used to remove plaque and to detect defects on the tooth surfaces as an adjunct to visual inspection. Teeth found to have occlusal pit and fissure sealants were classified as filled. Only teeth that were extracted due to caries were designating as missing. Permanent second molars were examined if their entire occlusal surface was erupted and could be examined; otherwise they were charted as unerupted. Clinical information included number of DMFS. We coded number of decayed surfaces as either less than or equal to 5 or great- er than 5 (the latter representing the upper third of the distribution). The number of missing teeth was negligible, so this information was not used in the analysis.

The Child Oral Health Impact Profile-Short Form (COHIP-SF) 19 [Broder et al., 2012b] has 19 items in three subscales: Oral Health, Functional Well-Being, and Socio-Emotional Well-Being. (1) Oral Health is comprised of specific oral symptoms that are not necessarily related to one another (e.g., pain, spots on teeth). (2) Functional Well-Being included items related to the child's ability to carry out specific everyday tasks or activities (e.g., speaking clearly, chewing). (3) Socio-Emotional Well-Being (Social-Emotional-School-Self) included items pertaining to peer interactions, mood states, school environment, and positive feelings about the self. All participants were instructed as follows: read carefully each statement and choose the answer that best describes you in the past 3 months regarding your teeth, mouth, or face. Responses were recorded as 'never' $=0$, 'almost nev$\mathrm{er}^{\prime}=1$, 'sometimes' $=2$, 'fairly often' $=3$, and 'almost all of the time' $=4$. Scoring of the negatively worded items was reversed. Higher COHIP-SF 19 scores reflect more positive OHRQoL, while lower scores reflect lower OHRQoL (see table 2 for COHIP-SF item details).

\section{Data Analysis}

Descriptive statistics for all measures were computed, including means and standard deviations (SD) for continuous and frequencies for categorical data. General Linear Modeling was used to compare the COHIP-SF scores for the decayed groups and separately for the filled groups on Oral Health, Functional Well-Being, Emotional WellBeing and the Overall COHIP-SF. All data manipulation and analyses were conducted with SAS 9.2, Cary, N.C., USA.

\section{Results}

Data relevant to these analyses were collected from 102 participants. Seven participants had an insufficient number of responses on the COHIP and were not included in these analyses. Average age of the participants was 12.8 $(\mathrm{SD}=1.2) ; 52.8 \%$ were female. Grade in school ranged from 6 to 10 (25.3\% 6th grade, $33.3 \% 7$ th, $29.9 \% 8$ th, $6.9 \%$ 9 th, and $4.6 \% 10$ th grade). Self-reported ethnicity was as follows: $53.9 \%$ Latino, $34.8 \%$ Black, $1 \%$ White, and $10 \%$ other.

Clinical Data. Decayed surfaces averaged $4.4(\mathrm{SD}=$ 3.9) with a wide range (0-17) and filled surfaces were similarly distributed with an average of $3.3(\mathrm{SD}=4.4)$, range $0-24$. DOI: $10.1159 / 000351693$
Genderson/Sischo/Markowitz/Fine/ Broder 
Table 2. Items in COHIP-SF

\section{Oral Health}

Had pain in your teeth/toothache

Had discolored teeth or spots on your teeth

Had crooked teeth or spaces between your teeth

Had bad breath

Had bleeding gums

Functional Well-Being

Had difficulty eating foods you would like to eat

Had trouble sleeping

Had difficultly saying certain words

Had difficulty keeping your teeth clean

Socio-Emotional Well-Being ${ }^{1}$

Been unhappy or sad

Felt worried or anxious

Avoided smiling or laughing with other children

Felt that you look different

Been worried about what other people think about your ...

Been teased, bullied or called names by other children

Missed school for any reason

Not wanted to speak/read out loud in class

Been confident

Felt that you were attractive (good looking)

${ }^{1}$ Questions finish with 'because of your teeth, mouth, or face'.

COHIP-SF. The average subscale scores were Oral Health 12.0 ( $\mathrm{SD}=4.7)$, Functional Well-Being 13.5 $(\mathrm{SD}=3.2)$, Socio-Emotional Well-Being 28.5 (6.7) and Overall COHIP-SF 57.5 (13.0).

COHIP-SF Scores by Clinical Severity. There were significant differences between the groups having fewer caries (as measured by the number of decayed surfaces) when compared to those having more caries on Oral Health $[\mathrm{F}(1,94)=6.1, \mathrm{p}<0.02]$, Functional Well-Being $[\mathrm{F}(1,94)=11.9, \mathrm{p}<0.001]$ and the Overall COHIP-SF $[\mathrm{F}(1,94)=7.3, \mathrm{p}<0.009]$. The scores on the Socio-Emotional Well-Being subscale were directionally consistent with those having more caries having lower OHRQoL. However, this result failed to reach conventional level of statistical significance $[\mathrm{F}(1,94)=3.5, \mathrm{p}<0.06]$. Post hoc power analysis suggests that our power to detect significant differences on the Socio-Emotional Well-Being subscale with the present effect size and sample size was fairly low (power $=0.68$ ) suggesting a larger sample may have yielded a $\mathrm{p}$ value less than 0.05 . Comparisons of COHIPSF scores for those having greater numbers of filled surfaces compared to lower number of filled surfaces revealed no significant differences on any subscale or the Overall COHIP-SF (data not shown). These findings are

Children's Oral Health-Related Quality of

Life Assessment consistent with other caries data and OHRQoL research [Varni et al., 2001; Locker and Allen, 2002; Broder and Wilson-Genderson, 2007].

\section{Clinically Meaningful Change versus Statistical Significance}

Another application of OHRQoL research involves changes in oral health as a result of specific treatment [Johnson, 2008]. In their recent study on the effect of fluoride varnish on caries among schoolchildren in rural Brazil, Arruda et al. [2011] conducted a double-blind, randomized, placebo-controlled trial with 379 children between 7 and 14 years who attended three schools in Brazil. Each school was visited four times at 6-month intervals when recruitment, dental exams, and fluoride varnish applications were completed. An interviewer-administered questionnaire was used to collect information from children regarding sociodemographic characteristics and oral health behavior (e.g., toothbrushing). Four calibrated dentists performed caries assessment exams using probes and mirrors (no radiographs) on permanent teeth only. Crude caries increments of decayed and filled surfaces (DFS) were compared between the fluoride varnish (5\% sodium fluoride) and the placebo group. After 12 months of follow-up, 210 participants completed the study. Those in the varnish group had significantly lower DFS increments than did those in the control group (10.8 vs. 13.3; $\mathrm{p}<0.01$ ), with PF of $40 \%$ (95\% CI: $34.3-45.7 \%$; $\mathrm{p}<1.01$ ). Thus, applications of $5 \% \mathrm{NaF}$ varnish are recommended as a public health measure in high-caries-risk populations. However, it is unknown if this statistically significant difference translates into clinically meaningful change for the children who participated in the study.

Including QoL assessment when evaluating clinical change begins to address our understanding of the qualitative impact the treatment has on the patient. In one study of preschool children with early childhood caries (ECC), changes in OHRQoL after treatment were reported. Using both patient and parental proxy assessments, Filstrup et al. [2003] found that children with ECC have significantly lower OHRQoL than their non-ECC counterparts. Further, they also reported that following dental treatment, those children with ECC showed significant improvement in their OHRQoL compared to their baseline measurements. Likewise, Huntington et al. [2011] found that children with ECC had significantly higher OHRQoL following surgical treatment at both their 3-month and 6-month follow-up appointments. In fact, 
by 6 months, the OHRQoL of children who had surgical treatment for the ECC was 'indistinguishable' from the study control group.

However, even statistical significance in QoL measures does not identify whether the change achieved has a qualitative impact on the patient [Tsakos et al., 2011]. Since a statistically significant change might not translate into real effects on patients' lives, there has been a shift in QoL outcomes research to measure clinically meaningful change or the Minimally Important Difference (MID) [Masood et al., 2012]. MID is defined as 'the smallest difference in score in the domain of interest which patients perceive as beneficial and which would mandate, in the absence of troublesome side effects and excessive cost, a change in the patient's management' [Jaeschke et al., 1989]. Instead of defining change on the basis of a statistical test of mean scores, MID uses the subjective perspective of the patient to determine what kind or how much change is meaningful to her/him [Juniper et al., 1994; Cella et al., 2002].

Two methods currently exist to measure MID: anchor methods and the standard error of measurement (SEM). Anchor methods use an independent standard (or 'anchor') that is interpretable and correlates at the 0.50 level (or higher) with the actual change score within the QoL instrument [Guyatt and Jaeschke, 1997; Cella et al., 2002]. They are intended to 'measure a patient's change score against clinically relevant or outside changes, such as expected changes caused by time, therapy, known disease diagnoses, or life events' [Wyrwich et al., 1999a]. Anchors divide subjects into groups that reflect no change, small positive changes, large positive changes, small negative changes, or large negative changes [Revicki et al., 2008]. While many different anchors could be chosen for the analysis, the best anchors are those that identify subjects who have changed 'to a small but meaningful degree' [Revicki et al., 2008]. Problems arise, however, when (1) retrospective self-reports, which are subject to recall bias, are used as anchors (as is most often the case), and (2) trying to establish the amount of change that is a 'reasonable indicator of minimal change' [Revicki et al., 2008]. These problems make anchor-based methods of determining the MID less than ideal.

Unlike anchor-based methods that rely on the use of an independent standard, SEM is a distribution-based method that is built upon the statistical properties of a study's results [Wyrwich et al., 1999b]. SEM is calculated based on the sample standard deviation and the sample reliability coefficient and is a function of the precision of the given instrument [Wyrwich et al., 1999b]. In other words, it is based on the estimate of error in an individual's score, which is inversely related to the reliability of a scale (i.e., the higher the SEM, the lower the scale reliability and vice versa). An important advantage of SEM is that it is relatively stable across populations with cutoffs based on confidence intervals. It also has the most potential for establishing benchmark scores that can be used to determine clinically meaningful change [Crosby et al., 2003].

Distribution-based indices such as SEM provide no direct information on the MID; instead, they establish a standardized metric for expressing an observed change [Revicki et al., 2008]. It is therefore important to verify the MID identified in a given instrument. One way to do this is by using a Global Assessment of Change scale. Our current ongoing longitudinal observational study of children undergoing secondary cleft surgery provides an example of using a global assessment scale to rate changes in OHRQoL. Participants were asked to rate changes in OHRQoL since their last clinic visit using a scale by domain [Juniper et al., 1994] and overall scale. Absolute global ratings were coded as follows: $1-3=$ minimal, 4-5 = moderate, and 6-7 = large clinically important change [Wyrwich et al., 1999a]. This global assessment, along with data from the COHIP, was used to determine the MID and clinically meaningful change. Specifically, we explored associations between OHRQoL and the Global Assessment of Change at the participants' followup visit. Means were compared using General Linear Modeling; analyses were conducted using SAS 9.2.

\section{Methods}

The sample is comprised of 384 school-aged Englishor Spanish-speaking children ages 7-19 with cleft who were followed for care at one of six geographically diverse cleft/craniofacial centers. These centers include: Children's Healthcare of Atlanta, Children's Hospital of Philadelphia, Lancaster Cleft Palate Clinic, New York University, University of Illinois at Chicago, and University of North Carolina-Chapel Hill. The sites also represent both rural and urban locations. As per IRB-approved protocols, data were collected at the children's regularly scheduled clinic visits with children and caregivers completing the COHIP packets and demographic information, respectively. Response rates across centers averaged 90\% (range: 78-95\%). (See earlier description of the COHIP.)

At the follow-up visit (approximately 1 year later), in addition to the other measures in the participants' packets, the Global Assessment of Change was administered. Participants were asked to rate changes in OHRQoL since their last clinic visit using a scale by domain [Juniper et
18

Caries Res 2013;47(suppl 1):13-21 DOI: $10.1159 / 000351693$
Genderson/Sischo/Markowitz/Fine/ Broder 
al., 1994] and overall scale (table 3). A response of zero on either scale represents no notable clinical change. Response ratings that capture important clinical change were coded as follows: minimal was comprised of ratings of 1 to 3 ( -3 to -1 and 1 to 3 ), moderate was comprised of ratings of 4 to 5 ( -4 to -5 and 4 to 5 ), and large was comprised of ratings of 6 to 7 ( -6 to -7 and 6 to 7 ) [Wyrwich et al., 1999a].

Data Analysis. Demographic data were computed (means and frequencies). Mean COHIP scores for the subscales and the Overall Total COHIP were compared for patients reporting no change, minimal, moderate, and large clinical change on Oral Health, Functional WellBeing, Self-Esteem and Total Change using General Linear Modeling with SAS 9.2.1.

\section{Results}

Youth participants averaged 12.6 years $(\mathrm{SD}=2.9) ; 42 \%$ were female. The sample's ethnic composition included: $68 \%$ White, $13.3 \%$ Latino or mixed, $8.2 \%$ Black or African American and $10.5 \%$ Asian. Fifty-four percent had private insurance.

COHIP subscale (mean, SD) scores were: Oral Health $(21.3,5.5)$, Functional Well-Being $(14.0,3.5)$, Emotional Well-Being (22.7, 4.0), School (9.9, 2.6), Self-Esteem $(21.6,3.7)$ and Overall COHIP $(89.5,13.4)$. Note that although the SEM approach for assessing clinically meaningful change does permit participants to endorse a decrement in OHRQoL, nearly none (3 total in the present sample) of these participants did. Thus, in all cases included in this analysis, ratings of change indicate either no change or improvement in OHRQoL. Distribution (in percent) of participants in the Global Assessment of Change groups representing no, minimal, moderate, and large amounts of change were: Oral Health $(32.4,32.1$, 18.4, 17.0), Functional Well-Being $(47.5,30.9,11.6,10.0)$, Self-Esteem $(50.1,27.9,10.3,11.6)$ and Overall change $(54.0,23.1,11.2,11.7)$ (table 4). There were significant differences on many COHIP subscales and overall for Oral Health, Functional Well-Being, Self-Esteem and Total ratings. Those with greater amounts of improvement, as represented by large clinical change, had higher scores on the COHIP as follows: global improvement in Oral Health symptoms was significantly associated with higher COHIP scores on Oral Health, School, Self-Esteem and Overall COHIP. Global improvement in Functional Well-Being was significantly associated with higher COHIP scores on Oral Health, Functional Well-Being, Emotional Well-Being and Overall COHIP. Global improvement in Self-Esteem was significantly associated

Children's Oral Health-Related Quality of Life Assessment
Table 3. COHIP Global Assessment Scale

\begin{tabular}{lc}
\hline No change (about the same) & 0 \\
\hline Almost the same, hardly any worse at all & -1 \\
A little worse & -2 \\
Somewhat worse & -3 \\
Moderately worse & -4 \\
A good deal worse & -5 \\
A great deal worse & -6 \\
A very great deal worse & -7 \\
\hline Almost the same, hardly any better at all & +1 \\
A little better & +2 \\
Somewhat better & +3 \\
Moderately better & +4 \\
A good deal better & +5 \\
A great deal better & +6 \\
A very great deal better & +7 \\
\hline
\end{tabular}

Table 4. COHIP Global Assessment of Change (GAC)

\begin{tabular}{lllll}
\hline COHIP GAC & $\begin{array}{l}\text { No } \\
\text { change }\end{array}$ & $\begin{array}{l}\text { Minimal } \\
\text { change }\end{array}$ & $\begin{array}{l}\text { Moderate } \\
\text { change }\end{array}$ & $\begin{array}{l}\text { Large } \\
\text { change }\end{array}$ \\
\hline Oral Health & $32.4 \%$ & $32.1 \%$ & $18.4 \%$ & $17.0 \%$ \\
Functional Well-Being & $47.5 \%$ & $30.9 \%$ & $11.6 \%$ & $10.0 \%$ \\
Self-Esteem & $50.1 \%$ & $27.9 \%$ & $10.3 \%$ & $11.6 \%$ \\
Overall & $54.0 \%$ & $23.1 \%$ & $11.2 \%$ & $11.7 \%$ \\
\hline
\end{tabular}

with higher COHIP scores on Functional Well-Being, Emotional Well-Being, School, Self-Esteem and Overall COHIP. Total global improvement was significantly associated with higher COHIP scores on Self-Esteem and Overall COHIP.

\section{Discussion}

COHIP scores reflect differences in OHRQoL among treatment-seeking patients based on the patients' reports of clinically meaningful change. Although these results are preliminary, they represent an important methodology rarely utilized in oral health research. Our future work will seek to explore the role of other patient and clinical characteristics in the context of these associations.

To date, one published oral health report has utilized this MID methodology in a caries clinical study [Malden et al., 2007]. Caregivers in New Zealand complet- 
ed the Parental-Caregiver Perceptions Questionnaire, an OHRQoL assessment, before their children had general anesthesia for dental caries and then completed a followup questionnaire again after 1-4 weeks (a global change rating scale was also included in the follow-up questionnaire). Comparisons between the baseline and follow-up data for the mean scores were examined and the MID was calculated for the overall scale and the subscales. The results revealed significant reductions in mean $\mathrm{Pa}$ rental-Caregiver Perceptions Questionnaire (improved OHRQoL) after treatment, with effect sizes ranging from moderate to large. Additionally, about two thirds of the children showed or exceeded the MID in their OHRQoL scores following their treatment.

Although studies in caries research that incorporate QoL outcomes are expanding [Sischo and Broder, 2011b], most studies report statistical difference using only change scores before and after treatment. Despite the widespread use of global change ratings in health-related QoL outcomes research across pediatric populations, its use in pediatric caries research is extremely limited.

\section{Conclusions}

Several published OHRQoL measures have been shown to have excellent psychometric properties and are suggested tools for oral health research. Currently, the gold standard in QoL research is to use school-aged children's subjective reports as youth can reliably complete standard self-administered, condition-specific question- naires [Feeny et al., 1998; Broder and Wilson-Genderson, 2007]. Reports have consistently shown modest yet significant correlations between clinical indices like dental decay with established OHRQoL instruments [Gherunpong et al., 2004; Locker et al., 2005; Broder et al., 2007; Huntington et al., 2011]. Although such measures are increasingly utilized in epidemiological as well as oral health clinical studies with pediatric populations, many clinical trials are not including these subjective evaluations in their research designs to measure outcomes [Sischo and Broder, 2011b]. Across patient groups in medicine, it is well documented that such QoL data often complement objective clinical data and may be useful in treatment decisions and measuring efficaciousness of care [Gherunpong et al., 2006]. Thus, during this era of evidence-based care coupled with reduced access to care among disadvantaged populations, utilizing OHRQoL assessments in epidemiological, observational, and clinical studies is recommended as an adjunct to dental caries research.

\section{Acknowledgments}

The authors thank the National Institute of Dental and Craniofacial Research for its support: DE0-18729 (H.L. Broder, PI) and DE-017968 (D. Fine, PI).

\section{Disclosure Statement}

The authors declare that they have no competing interests.

\section{References}

- Arruda AO, Senthamarai Kannan R, Inglehart MR, Rezende CT, Sohn W: Effect of 5\% fluoride varnish application on caries among school children in rural Brazil: a randomized controlled trial. Community Dent Oral Epidemiol 2012;40:267-276.

-Atchison KA, Shetty V, Belin TR, Der-Martirosian C, Leathers R, Black E, Wang J: Using patient self-report data to evaluate orofacial surgical outcomes. Community Dent Oral Epidemiol 2006;34:93-102.

Broder HL: Children's oral health-related quality of life. Community Dent Oral Epidemiol 2007;35(suppl 1):5-7.

Broder HL, McGrath D, Cisneros GJ: Questionnaire development: face validity and item impact testing of the Child Oral Health Impact Profile. Community Dent Oral Epidemiol 2007;35(suppl 1):8-19.
Broder HL, Wilson-Genderson M: Reliability and convergent and discriminant validity of the Child Oral Health Impact Profile (COHIP Child's version). Community Dent Oral Epidemiol 2007;35(suppl 1):20-31.

- Broder HL, Wilson-Genderson M, Sischo L: Health disparities among children with cleft. Am J Public Health 2012a;102:828-830.

Broder HL, Wilson-Genderson M, Sischo L: Reliability and validity testing for the Child Oral Health Impact Profile-Reduced (COHIP-SF 19). J Public Health Dent 2012b;72:302-312.

Cella D, Bullinger M, Scott C, Barofsky I; Clinical Significance Consensus Meeting Group: Group vs. individual approaches to understanding the clinical significance of differences or changes in quality of life. Mayo Clin Proc 2002;77:384-392.
Christie MJ, French D, Sowden A, West A: Development of child-centered disease-specific questionnaires for living with asthma. Psychosom Med 1993;55:541-548.

Crosby RD, Kolotkin RL, Williams GR: Defining clinically meaningful change in health-related quality of life. J Clin Epidemiol 2003;56:395407

DHHS, Oral Health in America: A report of the Surgeon General, US Department of Health and Human Services and National Institute of Dental and Craniofacial Research (eds). Rockville, National Institute of Health, 2000.

Feeny D, et al: Why not just ask the children?; in Drotar D (ed): Measuring Health-Related Quality of Life in Children and Adolescents. Hillsdale, Erlbaum, 1998, pp 171-187. 
Filstrup SL, Briskie D, da Fonseca M, Lawrence L, Jokovic A, Locker D, Stephens M, Kenny D, Wandera A, Inglehart MR: Early childhood caries and quality of life: child and parent perspectives. Pediatr Dent 2003;25:431-440.

Fine DH, Markowitz K, Furgang D, Fairlie K, Ferrandiz J, Nasri C, McKiernan M, Gunsolley J: Aggregatibacter actinomycetemcomitans and its relationship to initiation of localized aggressive periodontitis: longitudinal cohort study of initially healthy adolescents. J Clin Microbiol 2007;45:3859-3869.

-Gherunpong S, Sheiham A, Tsakos G: A sociodental approach to assessing children's oral health needs: integrating an oral health-related quality of life (OHRQoL) measure into oral health service planning. Bull World Health Organ 2006;84:36-42.

Gherunpong S, Tsakos G, Sheiham A: Developing and evaluating an oral health-related quality of life index for children; the CHILD-OIDP Community Dent Health 2004;21:161-169.

-Gregory J, Gibson B, Robinson PG: Variation and change in the meaning of oral health related quality of life: a 'grounded' systems approach. Soc Sci Med 2005;60:1859-1868.

Guyatt GH, Deyo DH, Charlson M, Levine MN, Mitchell A: Responsiveness and validity in health status measurement: a clarification. J Clin Epidemiol 1989;42:403-408.

Guyatt GH, Jaeschke RJ: Reassessing quality-oflife instruments in the evaluation of new drugs. Pharmacoeconomics 1997;12:621626.

Guyatt GH, et al: Measurements in clinical trials: choosing the right approach; in Spilker B (ed): Quality of Life and Pharmacoeconomics in Clinical Trials. Philadelphia, Lippincott-Raven, 1996, pp 41-48.

Huntington NL, Spetter D, Jones JA, Rich SE, Garcia RI, Spiro A 3rd: Development and validation of a measure of pediatric oral healthrelated quality of life: the POQL. J Public Health Dent 2011;71:185-193.

-Jaeschke R, Singer J, Guyatt GH: Measurement of health status: ascertaining the minimal clinically important difference. Control Clin Trials 1989;10:407-415.

-John MT, Hujoel P, Miglioretti DL, LeResche L, Koepsell TD, Micheelis W: Dimensions of oral-health-related quality of life. J Dent Res 2004;83:956-960.

-Johnson C: Outcome measures for research and clinical practice. J Manipulative Physiol Ther 2008;31:329-330. Tompson B, Guyatt G: Validity and reliability of a questionnaire for measuring child oralhealth-related quality of life. J Dent Res 2002; 81:459-463.

Juniper EF, Guyatt GH, Willan A, Griffith LE: Determining a minimal important change in a disease-specific Quality of Life Questionnaire. J Clin Epidemiol 1994;47:81-87.

Kimura A, Arakawa H, Noda K, Yamazaki S, Hara ES, Mino T, Matsuka Y, Mulligan R, Kuboki T: Response shift in oral health-related quality of life measurement in patients with partial edentulism. J Oral Rehabil 2012;39:44-54.

Locker D, Allen PF: Developing short-form measures of oral health-related quality of life. Public Health Dent 2002;62:13-20.

Locker D, Jokovic A, Tompson B: Health-related quality of life of children aged 11 to 14 years with orofacial conditions. Cleft Palate Craniofac J 2005;42:260-266.

Malden PE, Thomson WM, Jokovic A, Locker D: Changes in parent-assessed oral health-related quality of life among young children following dental treatment under general anaesthetic. Community Dent Oral Epidemiol 2008;36:108-117.

Markowitz K, Fairlie K, Ferrandiz J, Nasri-Heir C, Fine DH: A longitudinal study of occlusal caries in Newark New Jersey school children: relationship between initial dental finding and the development of new lesions. Arch Oral Biol 2012;57:1482-1490.

Masood M, Masood Y, Saub R, Newton JT: Need of minimal important difference for oral health-related quality of life measures. J Public Health Dent 2012, E-pub ahead of print.

McGrath C, Broder H, Wilson-Genderson M: Assessing the impact of oral health on the life quality of children: implications for research and practice. Community Dent Oral Epidemiol 2004;32:81-85.

Pahel BT, Rozier RG, Slade GD: Parental perceptions of children's oral health: the Early Childhood Oral Health Impact Scale (ECOHIS). Health Qual Life Outcomes 2007;5:6.

Reissmann DR, Remmler A, John MT, Schierz O, Hirsch C: Impact of response shift on the assessment of treatment effects using the Oral Health Impact Profile. Eur J Oral Sci 2012; 120:520-525.
Revicki D, Hays RD, Cella D, Sloan J: Recommended methods for determining responsiveness and minimally important differences for patient-reported outcomes. J Clin Epidemiol 2008;61:102-109.

Rumsey N, Harcourt D: Body image and disfigurement: issues and interventions. Body Image 2004;1:83-97.

Schwartz CE: Applications of response shift theory and methods to participation measurement: a brief history of a young field. Arch Phys Med Rehabil 2010;91(suppl):S38-S43.

Sischo L, Broder HL: Oral health-related quality of life: what, why, how, and future implications. J Dent Res 2011a;90:1264-1270.

$>$ Sischo L, Broder HL: Oral health-related quality of life: what, why, how, and future implications. J Dent Res 2011b;90:Appendix.

Tapsoba H, Deschamps JP, Leclercq MH: Factor analytic study of two questionnaires measuring oral health-related quality of life among children and adults in New Zealand, Germany and Poland. Qual Life Res 2000;9:559-569.

Tsakos G, Allen PF, Steele JG, Locker D: Interpreting oral health-related quality of life data. Community Dent Oral Epidemiol 2012;40: 193-200.

Varni JW, Seid M, Kurtin PS: PedsQL 4.0: reliability and validity of the Pediatric Quality of Life Inventory version 4.0 generic core scales in healthy and patient populations. Med Care 2001;39:800-812.

WHO: World Health Organization Constitution. Geneva, World Health Organization, 1948.

-WHOQOL: The World Health Organization Quality of Life assessment (WHOQOL): position paper from the World Health Organization. Soc Sci Med 1995;41:1403-1409.

Wyrwich KW, Nienaber NA, Tierney WM, Wolinsky FD: Linking clinical relevance and statistical significance in evaluating intra-individual changes in health-related quality of life. Med Care 1999a;37:469-478.

Wyrwich KW, Tierney WM, Wolinsky FD: Further evidence supporting an SEM-based criterion for identifying meaningful intra-individual changes in health-related quality of life. J Clin Epidemiol 1999b;52:861-873.

$>$ Yusuf H, Gherunpong S, Sheiham A, Tsakos G: Validation of an English version of the ChildOIDP index, an oral health-related quality of life measure for children. Health Qual Life Outcomes 2006;4:38. 